\title{
Multi-objective convex programming problem arising in multivariate sampling
}

\author{
Mohd. Vaseem Ismail ${ }^{1}$, Kaynat Nasser ${ }^{2}$, Qazi Shoeb Ahmad ${ }^{3 *}$ \\ ${ }^{1}$ Faculty of Pharmacy, Jamia Hamdard, New Delhi, INDIA \\ ${ }^{2}$ Department of Mathematics, Integral University, Lucknow, INDIA \\ ${ }^{3 *}$ Department of Mathematics, Integral University, Lucknow, INDIA \\ "Corresponding Author: e-mail: qazishoeb@rediffmail.com
}

\begin{abstract}
In this paper, we formulate the multivariate allocation problem as a multi-objective convex programming problem. The objective functions are convex and there is a single linear constraint with some upper and lower bounds. We also consider a two dimensional multivariate problem when the cost is minimized. A numerical example is given to illustrate the solution procedure.
\end{abstract}

Keywords: Multivariate stratified sampling, convex programming, multi-objective problem

\section{Introduction}

In multivariate surveys, there are more than one population characteristics to be estimated and usually these characteristics are of conflicting nature. The derivation of the optimal sample numbers among various strata or various stages thus requires some special treatment. However, although the consideration of multiple objectives may seem a novel concept, virtually any nontrivial, real world problem invariably involves multiple objectives. For example, the success of an airplane is determined by such things as its cost (to be minimized), payload (to be maximized), speed (to be maximized), maximum range (to be maximized), weight (to be minimized), survivability (to be maximized) etc. And, in the design of an aircraft, we may actually hope to optimize each and every one of these parameters. The importance of multi-objective optimization can also be seen by the large number of applications presented in the literature as Agrell et al. (1998), Armann (1989), Eschenauer (1988), Ferreira and Machado (1996), Fu et al. (2000), Fu and Diwekar (2004), Johnson and Diwekar (2001), Kumar and Tewari (1991), Miettinen (1999), Ohkubo, Dissanayake and Taniwaki (1998). Most of these applications are multi-objective problems of nonlinear nature, which is why we need tools for nonlinear programming capable of handling multiple conflicting or incommensurable objectives.

\section{Multivariate stratified sampling}

We consider a multivariate population partitioned into $L$ strata. Suppose that p characteristics are measured on each unit of the population. We assume that the strata boundaries are fixed in advance. Let $n_{i}$ be the number of units drawn without replacement from $i^{\text {th }}$ stratum $(i=1,2, \ldots, L)$. Let $N_{i}$ be the size of $i^{\text {th }}$ stratum. For $j^{\text {th }}$ character, an unbiased estimate of the population mean $\bar{Y}_{j}(j=1,2, \ldots, p)$, denoted by $\bar{y}_{j s t}$, has its sampling variance

$$
V\left(\bar{y}_{j s t}\right)=\sum_{i=1}^{L}\left(\frac{1}{n_{i}}-\frac{1}{N_{i}}\right) W_{i}^{2} S_{i j}^{2},(j=1,2, \ldots, p)
$$




$$
W_{i}=\frac{N_{i}}{N}, \quad S_{i j}^{2}=\frac{1}{N_{i}-1} \sum_{h=1}^{N_{i}}\left(y_{i j h}-\bar{Y}_{i j}\right)^{2}
$$

Substituting $a_{i j}=W_{i}^{2} S_{i j}^{2}$, we get

$$
V\left(\bar{y}_{j s t}\right)=\sum_{i=1}^{L} \frac{a_{i j}}{n_{i}}-\sum_{i=1}^{L} \frac{a_{i j}}{N_{i}},(j=1,2, \ldots, p)
$$

Let $C_{i j}$ be the cost of enumerating the $j^{\text {th }}$ character in the $i^{\text {th }}$ stratum and let $C$ be the upper limit on the total cost of the survey. Then assuming linear cost function, one should have

$$
\sum_{i=1}^{L} \sum_{j=1}^{p} C_{i j} n_{i} \leq C \text { or } \sum_{i=1}^{L} C_{i} n_{i} \leq C
$$

Where $C_{i}=\sum_{j=1}^{p} C_{i j}$, the cost of enumeration of all the $p$ characters in the $i^{\text {th }}$ stratum.

Further one should have

$$
1 \leq n_{i} \leq N_{i},(i=1,2, \ldots, L)
$$

We determine, the optimum values of $n_{i}$ by minimizing (in some sense) all the $p$ variances (equation 1 ) for a fixed budget (equation 2) i.e. we have to

$$
\begin{aligned}
& \text { Minimize } V_{j}=\sum_{i=1}^{L} \frac{a_{i j}}{n_{i}}-\sum_{i=1}^{L} \frac{a_{i j}}{N_{i}},(j=1,2, \ldots, p) \\
& \text { Subject to } \sum_{i=1}^{L} C_{i} n_{i} \leq C \\
& \text { and } \quad 1 \leq n_{i} \leq N_{i}, \quad(i=1,2, \ldots, L)
\end{aligned}
$$

Since $N_{i}^{s}$ are given, it is enough to minimize

$$
V_{j}=\sum_{i=1}^{L} \frac{a_{i j}}{n_{i}},(j=1,2, \ldots, p)
$$

Using $X_{i}$ for $n_{i}$, the problem (equation 4) can be written as the following multi-objective non-linear programming problem:

$$
\begin{array}{ll}
\text { Minimize } V_{j}=\sum_{i=1}^{L} \frac{a_{i j}}{X_{i}}, \quad(j=1,2, \ldots, p) & (a) \\
\text { Subject to } \quad \sum_{i=1}^{L} C_{i} X_{i} \leq C & (b) \\
\text { and } & 1 \leq X_{i} \leq N_{i}, \quad(i=1,2, \ldots, L)
\end{array}
$$

The objective functions in (equation 5) are convex [see Kokan and Khan (1967)], the single constraint is linear and the bounds are also linear. The problem (5) is, therefore a multi-objective convex programming problem.

If some tolerance limits, say $v_{j}$ are given on variances of the $p$ characters then the allocation problem reduces to the single objective convex programming problem as:

$$
\begin{array}{ll}
\text { Minimize } & \sum_{i=1}^{L} C_{i} X_{i} \\
\text { Subject to } & \sum_{i=1}^{L} \frac{a_{i j}}{X_{i}} \leq v_{j}, \quad(j=1,2, \ldots, p) \\
\text { and } & 1 \leq X_{i} \leq N_{i},(i=1,2, \ldots, L)
\end{array}
$$




\section{Solution of a two dimensional multivariate problem when the cost is minimized}

Let us consider the problem (equation 6). Due to its special character (only two dimension), we give in the following an easy method of solution by using the analytical approach of Kokan and Khan (1967). The problem is to

$$
\begin{array}{ll}
\text { Minimize } & C=\sum_{i=1}^{2} C_{i} X_{i} \\
\text { Subject to } & \sum_{i=1}^{2} \frac{a_{i j}}{X_{i}} \leq v_{j}, \quad(j=1,2, \ldots, p) \\
\text { and } \quad & 1 \leq X_{i} \leq N_{i}, \quad(i=1,2)
\end{array}
$$

Using the transformation $X_{i}=\frac{1}{X_{i}}$, this reduces to

$$
\begin{aligned}
& \text { Minimize } C=\sum_{i=1}^{2} \frac{C_{i}}{x_{i}} \\
& \text { Subject to } \\
& \sum_{i=1}^{2} a_{i j} x_{i} \leq v_{j}, \quad(j=1,2, \ldots, p) \\
& \text { and } \quad \frac{1}{N_{i}} \leq x_{i} \leq 1, \quad(i=1,2)
\end{aligned}
$$

\section{Solution procedure}

First we identify the linear constraints $k_{1}$ and $k_{2}$ such that

$$
\left.\begin{array}{l}
\min _{j} \frac{v_{j}}{a_{1 j}}=\frac{v_{k 1}}{a_{1 k 1}} \\
\min _{j} \frac{v_{j}}{a_{2 j}}=\frac{v_{k 2}}{a_{2 k 2}}
\end{array}\right\}
$$

Let us denote the minimum of $C$ subject to the constraint $(j)$ by $\underline{x}^{(j)}$. An explicit expression for $\underline{x}^{(j)}=\left(x_{1}^{(j)}, x_{2}^{(j)}\right)$ is given by

$$
x_{i}^{(j)}=\frac{v_{j} \sqrt{a_{i j} C_{i}}}{a_{i j}\left\{\sum_{i=1}^{2} \sqrt{a_{i j} C_{i}}\right\}}, \quad(i=1,2)
$$

We illustrate the method by an (hypothetical) example represented in the following Figure 1 in which we have taken four constraints. The level curves of the objective functions touching the various constraints are also traced.

The minimum intercept on $x_{1}$ is cut by the constraint (1) and the minimum intercept on $x_{2}$ is cut by the constraint (4).

Now $\underline{x}^{(4)}$ violates the constraint (1) and $\underline{x}^{(1)}$ violates the constraint (4). A dangling solution, will then be the point of intersection of the lines (1) and (4), viz $\underline{x}^{(1,4)}$.

This new point however violates the constraint (2). So we test $\underline{x}^{(2)}$, which violates the constraint (1). Since $\underline{x}^{(1)}$ also violates the constraint (2), the intersection of the lines (1) and (2) is tested, which satisfies all the constraints and thus gives the optimal solution. 


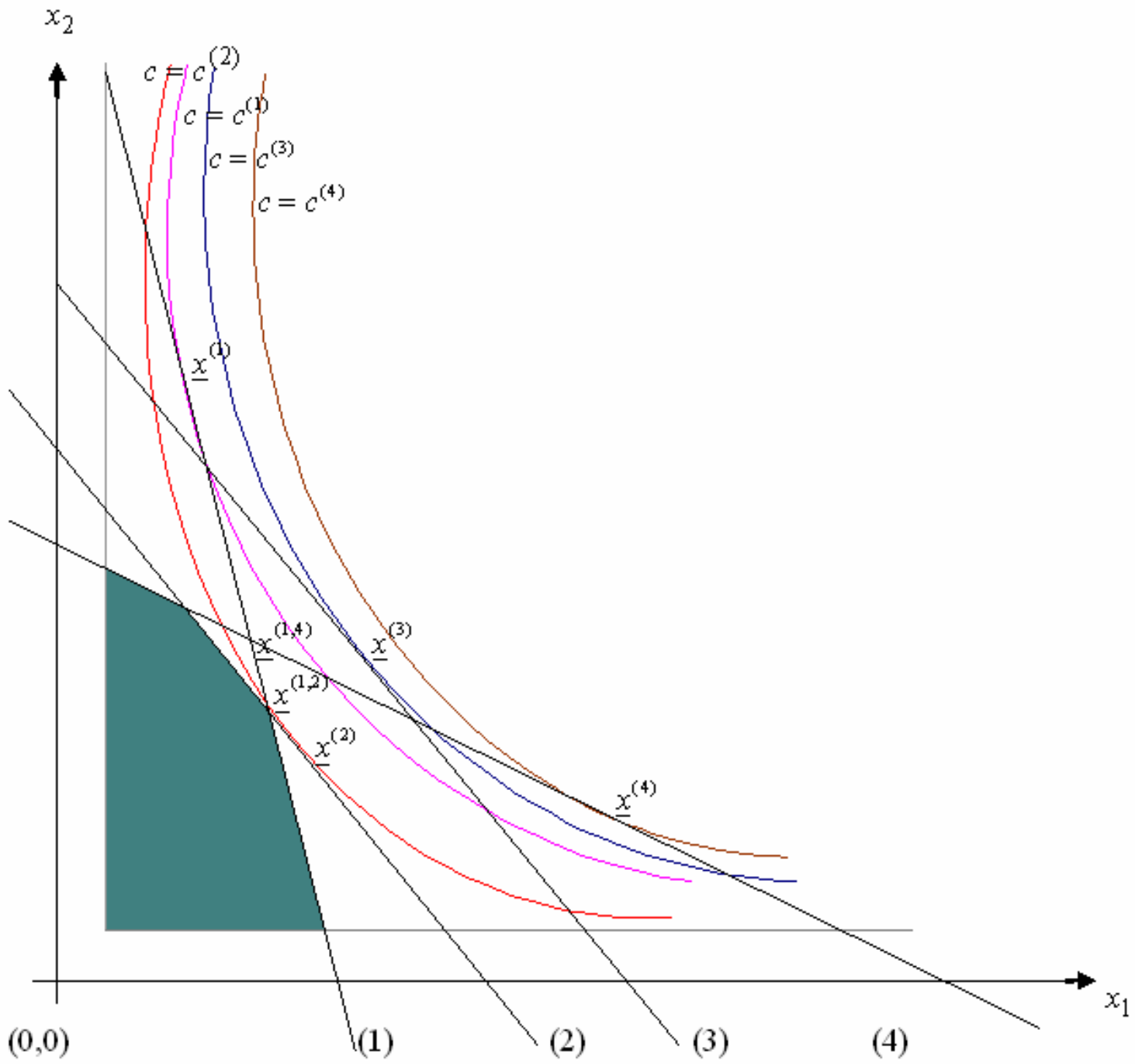

\section{Numerical example}

Figure 1. Graph for the hypothetical example

Let us consider the numerical example in which the upper bounds on three variances are given as $0.30,0.60$ and 0.50 respectively, divided into two strata with three characters under study for which the values of $N_{i}, W_{i}, S_{i 1}, S_{i 2}, S_{i 3}, C_{i 1}, C_{i 2}$ and $C_{i 3}$ are given in the following table:

\begin{tabular}{|c|c|c|c|c|c|c|c|c|}
\hline Stratum $(i)$ & $N_{i}$ & $W_{i}$ & $S_{i 1}$ & $S_{i 2}$ & $S_{i 3}$ & $C_{i 1}$ & $C_{i 2}$ & $C_{i 3}$ \\
\hline 1 & 180 & 0.40 & 1.5 & 2.25 & 0.75 & 0.6 & 0.9 & 1.5 \\
\hline 2 & 270 & 0.60 & 3.0 & 4.75 & 5.25 & 0.8 & 1.2 & 2.0 \\
\hline
\end{tabular}

The variance coefficients matrix is obtained by $a_{i j}=W_{i}^{2} S_{i j}^{2}$ as

$$
\left(a_{i j}\right)=\left(\begin{array}{lll}
0.36 & 0.81 & 0.09 \\
3.24 & 8.12 & 9.92
\end{array}\right)
$$

Let us fix the budget at 100 units. 
Then the problem is to be solved is

$$
\begin{array}{cl}
\text { Minimize } & \frac{3}{x_{1}}+\frac{4}{x_{2}} \\
\text { Subjectto } & 0.36 x_{1}+3.24 x_{2} \leq 0.30 \\
& 0.81 x_{1}+8.12 x_{2} \leq 0.60 \\
& 0.09 x_{1}+9.92 x_{2} \leq 0.50 \\
& 0.0056 \leq x_{1} \leq 1 \\
& 0.0037 \leq x_{2} \leq 1
\end{array}
$$

We identify the linear constraints (2) and (3) by using (9).

By using (9), we obtain $\underline{x}^{(2)}$ and $\underline{x}^{(3)}$ as $(0.1591,0.0580)$ and $(0.4233,0.0466)$.

Now, $\underline{x}^{(3)}$ violates the constraint (2) and $\underline{x}^{(2)}$ violates the constraint (3). Then the solution is $\underline{x}^{(2,3)}=(0.2585,0.0481)$. This point also satisfies the constraint (1). Hence it is an optimal solution to the given problem.

The values of sample sizes $n_{1}$ and $n_{2}$ are found respectively as 3.87 and 20.79 which rounded to the nearest integers are 4 and 21 . The value of the objective function at optimal point is 96.

\section{Conclusions}

The paper dealt with an important problem in multiobjective non linear programming for optimal allocation in stratified sampling, including various formulations of the problem, identifying the linear constraints, tracing the level curves of the objective functions touching the various constraints and then finding optimal solution which satisfies all the constraints. We considered non-linear cost function and continuous and integer sample size variables, graphical approach to easily understand the problem and later on illustrated by numerical example. Further studies may consider both the objective function and constraints as non-linear function and represent it graphically.

\section{References}

Agrell, P.J., B.J. Lence, and A. Stam, 1998. An interactive multicriteria decision model for multipurpose reservoir management: the Shellmouth reservoir. Journal of Multi-Criteria Decision Analysis, Vol. 7, No. 2, pp. 61-86.

Aoyama, H., 1963. Stratified random sampling with optimum allocation for multivariate populations. Ann. Inst. Stat. Math., Vol. 14, pp. 251-258.

Armann, R., 1989. Solving multiobjective programming problems by discrete optimization. Optimization, Vol. 20 , pp. $483-492$.

Bethel, J., 1985. An optimum allocation algorithm for multivariate survey. Proceedings of the Survey Research Section, ASA, pp. 204-212.

Chatterjee, S., 1968. Multivariate stratified surveys. Jour. Amer. Stat. Assoc. Vol. 63, pp. 530-534.

Chromy, J.R., 1987. Design optimization with multiple objectives. Proceedings of the Survey Research Section, ASA, pp. $194-199$.

Cochran, W.G., 1977. Sampling Techniques. John Wiley and Sons, New York.

Eschenauer, H.A., 1988. Multicriteria optimization techniques for highly accurate focusing systems In W. Stadler (ed.), Multicriteria Optimization in Engineering and in the Sciences, pp. 309-354. New York: Plenum Press.

Ferreira, P.A.V. and M.E.S. Machado, 1996. Solving multiple-objective problems in the objective space. Journal of Optimization Theory and Applications, Vol. 89, No. 3, pp. 659-680.

Fu, Y., U.M. Diwekar, D. Young, and H. Cabezas, 2000. Process design for environment: a multiobjective framework under uncertainty. Journal of Clean Products and Processes Vol. 2, pp. 92-105.

Fu, Yan and Diwekar, U.M., 2004. An efficient sampling approach to multiobjective optimization. Annals of Operation Research., Vol. 132, pp. 109-134.

Ghosh, S. P., 1958. A note on stratified random sampling with multiple characters. Cal. Stat. Assoc. Bull., Vol. 8, pp. 81-89.

Ismail, M.V., and Razzaq, A., 2004. Chebyshev solution to a multivariate stratified sampling problem. Pure and Applied Mathematika Sciences. LIX, pp. 1-2.

Johnson, T.L. and U.M. Diwekar, 2001. Hanford waste blending and the value of research: stochastic optimization as a policy tool. Journal of Multi-Criteria Decision Analysis Vol. 10, pp. 87-98.

Kelly, J.E., 1960. The cutting plane method for solving convex programs. Jour. Soc. Indust. Appl. Math. Vol. 8, pp. 703-712.

Kokan, A.R and Khan, S.U., 1967. Optimum allocation in multivariate surveys-an analytical solution. Jour. Roy. Stat. Soc. Ser. B.29, pp. 115-125. 
Kumar, P., N. Singh, and N.K. Tewari, 1991. A nonlinear goal programming model for multistage, multiobjective decision problems with application to grouping and loading problem in a flexible manufacturing system. European Journal of Operational Research, Vol. 53, No. 2, pp. 166-171.

Miettinen, K.M., 1999. Nonlinear Multiobjective Optimization. Norwell, MA: Kluwer Academic.

Neyman, J., 1934. On the two different aspects of representative method: the method of stratified sampling and the method of purposive selection. Journal of Royal Stastistical Society, Vol. 97, pp. 558-606.

Ohkubo, S., P.B.R. Dissanayake, and K. Taniwaki, 1998. An approach to multicriteria fuzzy optimization of a prestressed concrete bridge system considering cost and aesthetic feeling. Structural Optimization Vol. 15, No. 2, pp. 132-140.

Yates, F., 1960. Sampling Methods for Censuses and Surveys ( $2^{\text {nd }}$ ed). Charles Griffin and Co. Ltd. London.

\section{Biographical notes}

Dr. Mohd.Vaseem Ismail received M.Sc. and Ph.D. in Statistics from Aligarh Muslim University, Aligarh, India. He is an Assistant Professor in the Faculty of Pharmacy, Jamia Hamdard, New Delhi, India. He has more than six years of experience in teaching and research. His research interests include optimization in sampling and fuzzy programming. He has published five research papers in referred national and international journals. He has also presented two research articles in national and international conferences. He has written five books related to Bio-Statistics and Mathematics.

Kaynat Nasser received M.Sc. and M.Phil. in Statistics from Aligarh Muslim University, Aligarh, India. Presently she is pursuing Ph.D. from the Department of Mathematics, Integral University, Lucknow.

Dr. Qazi Shoeb Ahmad received M.Sc. and Ph.D. in Operations Resaerch from Aligarh Muslim University, Aligarh, India He is an Assistant Professor in the Department of Mathematics, Integral University, Lucknow, India. He has more than 10 years of experience in teaching and research. His current area of research includes Integer Programming, Sequencing and Mathematical Programming in Sampling. He has published fifteen research papers in referred national and international journals. He has also presented three research articles in national and international conferences. He has written seven books related to Mathematics and Statistics.

Received March 2010

Accepted November 2010

Final acceptance in revised form November 2010 